\title{
THE BENEFITS OF INCLINED-ORBIT OPERATIONS FOR GEOSTATIONARY ORBIT COMMUNICATION SATELLITES
}

\author{
Lihua Ma \\ National Astronomical Observatories, Chinese Academy of Sciences, Beijing 100012, China \\ e-mail: mlh@nao.cas.cn
}

\begin{abstract}
Geostationary orbit (GEO) communication satellites can be extended in lifetime by switching to inclined-orbit operations. In this mode, a small amount of propellant is reserved to maintain the assigned orbit longitude. Inclination is allowed to build up at a rate of approximately $0.8^{\circ}$ per year. Developing these space resources can bring out a number of benefits. Besides communication application, these satellites can be used to construct navigation constellation of the Chinese Area Positioning System (CAPS). In this present paper, the realization way of communication and navigation applications is studied and the benefits and problems are explained.
\end{abstract}

Keywords: Chinese Area Positioning System (CAPS); geostationary orbit satellite; inclinedorbit operations; communication application; navigation application

\section{INTRODUCTION}

The main source of perturbation force for geostationary orbit (GEO) is combined gravitational attractions of the sun and the moon, which causes orbital inclination to increase by about $0.75^{\circ}-0.95^{\circ}$ per year. This is countered by north-south station-keeping (NSSK) maneuvers so as to keep the GEO satellite within a small scale of the equatorial plane under the International Telecommunication Union (ITU) regulations. Additionally, the bulge of the earth causes a longitudinal drift, which is compensated by east-west station-keeping (EWSK) maneuvers. Finally, solar radiation pressure caused by the transfer of momentum from the sun's light and infrared radiation both flattens the orbit and disturbs the orientation of the satellite. The orbit is compensated by an eccentricity control maneuver that can sometimes be combined with EWSK, whereas satellite's orientation is maintained by momentum wheels supplemented by magnetic torquers and thrusters (Agrawal, 1986; Zhang, 1998). All these maneuvers consume on-board propellant. However, it is crucial for GEO satellites to have the most fuel-efficient propulsion operations in order to save weight of a satellite. Considering that the demands for EWSK maneuvers require only about 10 percent of propellant needed to correct for NSSK excursions, operational lifetime of the GEO satellite can been extended by ceasing NSSK and providing only EWSK corrections (known inclined-orbit operations). This results in the satellite moving to higher latitude and lower latitude in an S-shaped curve. The satellite is thus moving both below and above the equatorial plane in a very regular figure-8like motion as seen from the ground, the inclination increases gradually with time and the satellite becomes a slightly inclined geosynchronous orbit (SIGSO) satellite (Atia et al., 1990; Bauer et al., 2002; Shi et al., 2009; Ma et al., 2011). 
The Chinese Area Positioning System (CAPS) is an area positioning system based on GEO communication satellites. Navigation and ranging signals are continuously generated at a ground station and retransmitted by the transponder on GEO communication satellite. Combining with barometric altimetry technique the user realizes navigation and positioning in the experimental verification phase of CAPS (Ai, et al., 2008, 2009). Several CAPS satellites have been executed the inclined-orbit operations and become SIGSO satellites. Besides navigation applications, signal transponders on these SIGSO satellites continue to perform communication function (Ai et al., 2008; Ai et al., 2009; Shi et al., 2009).

In this paper we study the benefits of inclined-orbit operations for GEO communication satellites. Section 2 and section 3 describe some problems and give resolving ways during communication and navigation applications in CAPS respectively. The final section presents a summary of the findings of this paper.

\section{COMMUNICATION APPLICATION}

\subsection{Satellite downlink beam coverage}

As mentioned above, when NSSK of a geostationary satellite is relaxed, the orbit of the satellite becomes inclined with an inclination that increases gradually with time. Downlink beam coverage of on-board antenna is subjected to the figure-8-like orbit place of the SIGSO satellite. Periodic change exists in the edge of antenna beam coverage even if the communication satellite adopts global beam antenna. Fig. 1 shows the increase in area of the earth's surface that will have direct line-of-sight to the APSTAR 1 satellite that is nominally located at $\left(142^{\circ} \mathrm{E}, 0\right)$ if that satellite is drifted into an inclination with $5^{\circ}$. The shaded area represents the extra illumination of the earth. It is obvious that the majority of this new coverage falls in the polar region.

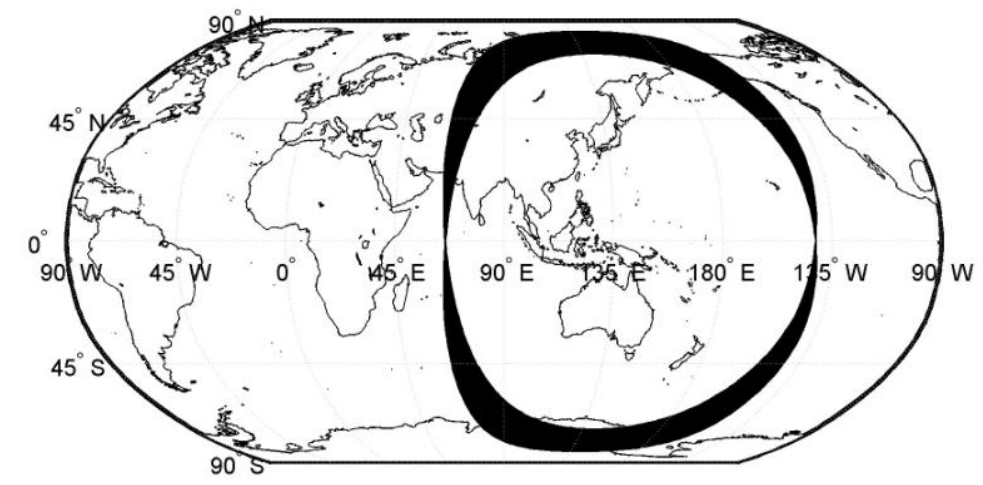

Fig.1 Ground coverage of antenna beam for the APSTAR 1 satellite with $5^{\circ}$ inclination

In order to overcome the coverage change of on-board antenna from inclination angle, pointing equipments including software and hardware should be continuously adjusted according to the space place of the satellite. Given that the user mainly locates in the center of antenna beam coverage, far away from the edge of beam coverage, as a simplification way, antenna pointing can be maintained at the average region so as to satisfy communication requirements of ground coverage area.

\subsection{Satellite tracking antenna for ground station}

Azimuth and elevation angles from ground station to the SIGSO satellite change with time in 24-hour period. As for orbit place at special time, one can calculate azimuth and elevation angles. In order to track the satellite, the pointing of ground antenna should be adjusted in certain scale, corresponding to variation scale of azimuth and elevation angles. Namely the antenna pointing orientation should be within a small box from the two angles. Supposed that the APSTAR 1 satellite has been in SIGSO orbit with $5^{\circ}$ inclination, the tracking range of azimuth and elevation angles is contoured in Fig. 2. 

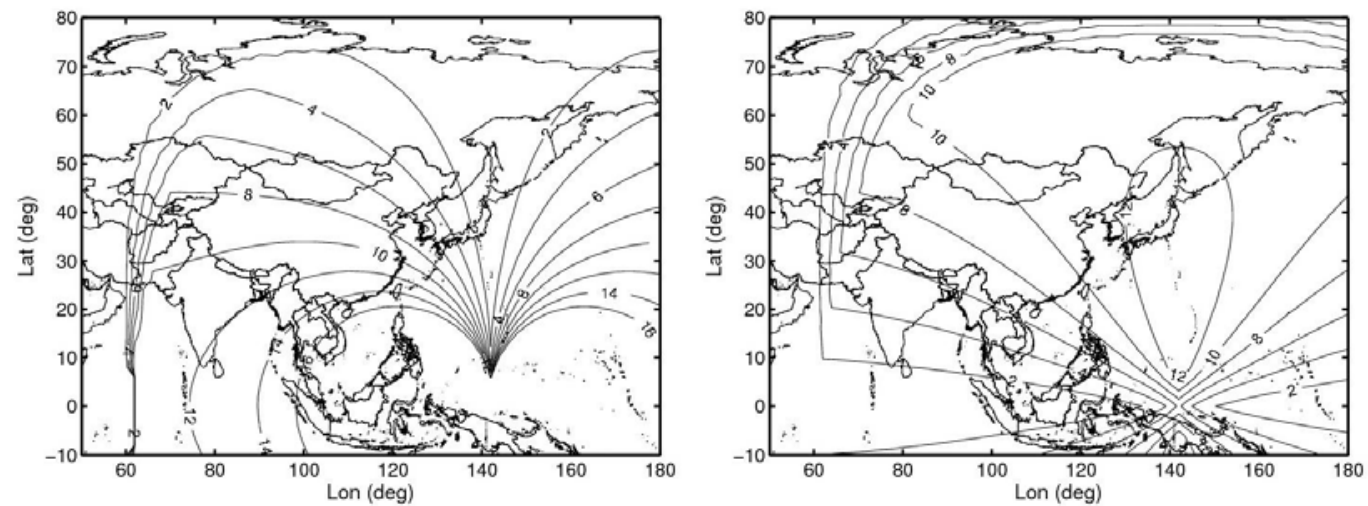

Fig. 2 The tracking range of azimuth and elevation angles for the APSTAR 1 satellite with $5^{\circ}$ inclination

The automatic gain control (AGC) system monitors the level of the received signal, and moves the antenna periodically to peak the signal so that ground antenna can track the satellite. With NORAD two-line element (TLE) the satellite coordinate can be easily calculated. Furthermore the azimuth and elevation angles can be obtained. Therefore, as an alternative method, program-control mathematically calculates the pointing angles to the satellite and moves the antenna accordingly. Calculations based on program are entered into the controller of tracking antenna for ground station.

\subsection{Polarization loss}

For an antenna is a transducer that converts radio frequency electric current to electromagnetic waves that are then radiated into space, antenna polarization is an important consideration when selecting and installing antennas. Most wireless communication systems including satellite communication use either linear (vertical, horizontal) or circular polarization. Besides from above pointing loss, as for linear polarization antenna equipped on these SIGSO communication satellites, polarization mismatch can result in remarkable signal attenuation. Taking the APSTAR 1 satellite with $5^{\circ}$ inclination as example, polarization loss in coverage region is contoured in Fig. 3 if polarization of user terminals has been regulated in Beijing ground station (Ma et al., 2010).

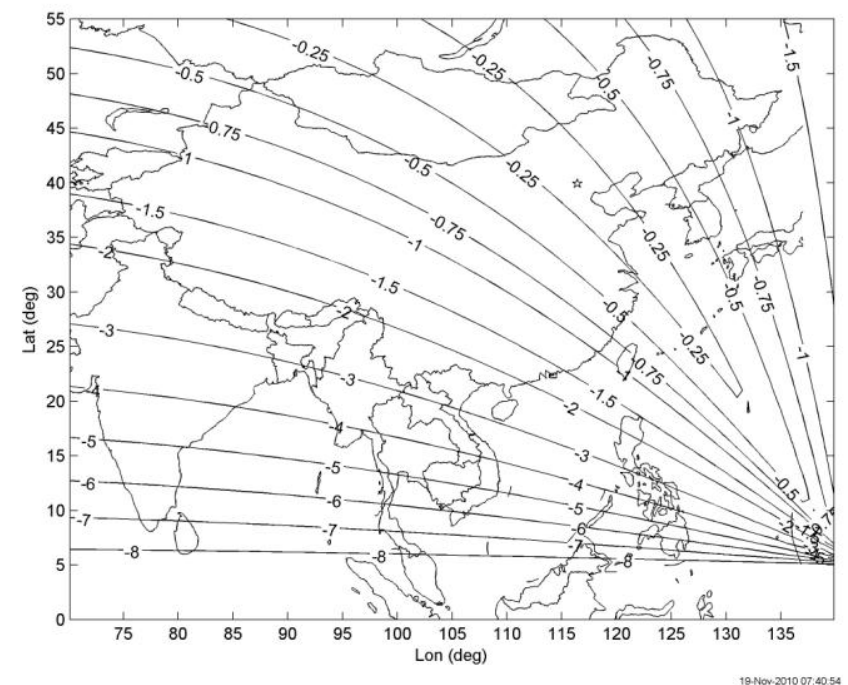

Fig. 3 Polarization loss of the APSTAR 1 satellite with $5^{\circ}$ inclination 
It's can be seen that polarization regulation on ground antenna should be done in a specific region in order to improve communication quality of the SIGSO satellite. Of course, the regulation is unnecessary if the GEO satellite antenna adopts circular-polarization mode.

\section{NAVIGATION APPLICATION}

\subsection{Doppler frequency drift}

The Doppler frequency shift is the change in frequency of a wave for an observer moving relative to the source of the wave, and occurs due to the variation in relative velocity between the earth and the satellite. Because of inclination, relative velocity existing in the user and the SIGSO satellite results in obvious Doppler frequency drift. When the SIGSO inclination is about $3^{\circ}$, theoretical value of the Doppler shift is about $211 \mathrm{kHz}$ if the downlink signal adopts C-band carrier (Li and Hao, 1996). Navigation function of the SIGSO satellite is influenced by the shift making a little effect on communication function. In the CAPS navigation, velocity measurements should be made by carrier phase measurements which estimate the Doppler frequency of the received satellite signals. Here the frequency pre-bias technique is performed and corresponding principle is shown in Fig. 4 (Wu, et al., 2009).

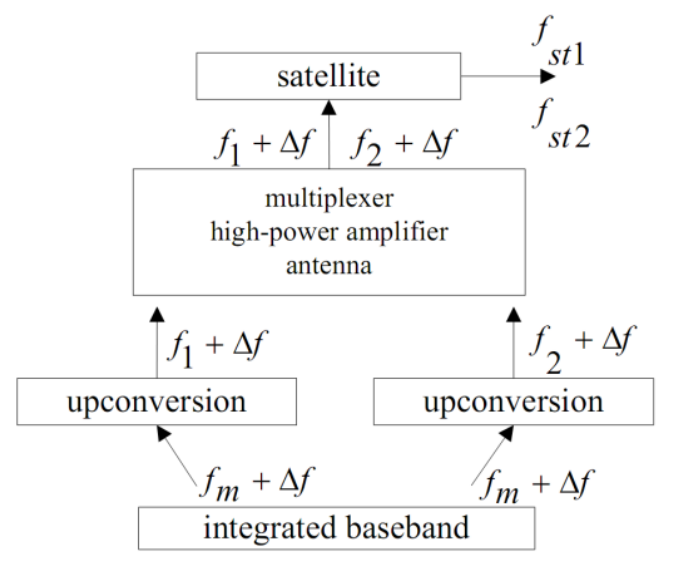

Fig.4 The principle of frequency pre-bias

The pre-bias frequency $\Delta f$ effecting on two output intermediate frequency $f_{m}$ is calculated in the integrated baseband. The frequencies of carriers $C_{1}$ and $C_{2}$ become $f_{1}+\Delta f$ and $f_{2}+\Delta f$ respectively after adopting different upconversion devices. The two carriers are transmitted to the satellite passing through multiplexer device, high-power amplifier and antenna. Finally the frequency received by the satellite should add the Doppler frequency derived from the ground to the satellite.

\subsection{PDOP with altimeter aiding}

Spatial configuration of navigation constellation pays an important role in positioning performance. Constellation configuration can be represented as position dilution of precision (PDOP) factor. Position accuracy is derived from pseudorange measurement error magnified the PDOP value (Kaplan and Hegarty, 2006). Thereafter we analyses the effects of SIGSO satellites on navigation performance, with emphasis on investigating PDOP optimization extent from SIGSO satellites.

Recently, the CHINASTAR-1, SINOSAT-1, APSTAR-1A and APSTAR-1 satellites nominally located at $\left(87.5^{\circ} \mathrm{E}, 0\right),\left(110.5^{\circ} \mathrm{E}, 0\right),\left(130^{\circ} \mathrm{E}, 0\right)$ and $\left(142^{\circ} \mathrm{E}, 0\right)$ are adopted in the constellation of CAPS. Among these satellites, the APSTAR 1A and APSTAR 1 satellites have been regulated into SIGSO satellites under inclined-orbit operations. As mentioned above, the altimeter technique has been applied in CAPS. Some studies gave efficient DOP calculation with altimeter aiding (Jwo, 2001; Han et al., 2009; Ma et al., 2011). In order to 
clearly display the PDOP value, we set 6 ground observation stations including Beijing, Shanghai, Changchun, Kunming, Ulumuqi and Xiaan in Mainland China. Supposed that satellites can always receive uplink navigation signals during each 24-hour period, the mask degree of the user receiver is given as $5^{\circ}$. According to theory on celestial mechanics, the time interval of SIGSO satellites through the equator is corresponding to longitude difference. Therefore, given the time of some SIGSO satellite going through the equator, the time of other SIGSO satellites going through the equator are determined. Among four CAPS satellites, $\mathrm{n}(\mathrm{n}=0,1,2,3,4)$ satellites become SIGSO satellites and corresponding daily PDOP change in every station is plotted in Fig. 5.
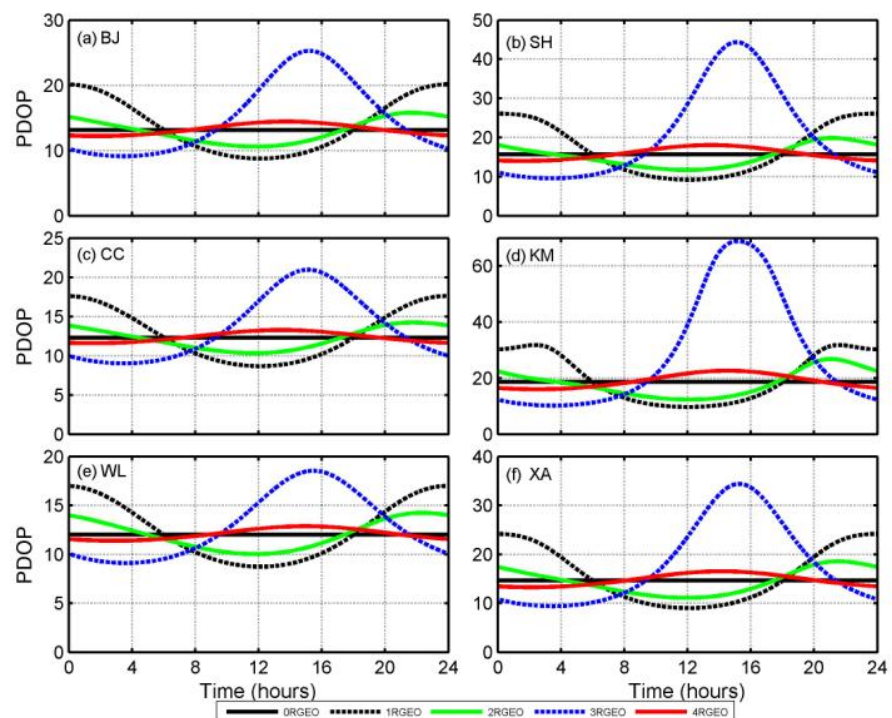

Fig. 5 Daily PDOP change of constellation with n SIGSO satellites

$$
(\mathrm{n}=0,1,2,3,4) \text { with altimeter aiding }
$$

As can be seen, compared with constellation with all GEO satellites, it is obvious that SIGSO satellites can improve CAPS performance in some time period, however, the PDOP value becomes worse in other time period. Meanwhile daily PDOP change with 2 SIGSO satellites is close to that with 4 SIGSO satellites, and daily PDOP change with 1 SIGSO satellites is close to that with 3 SIGSO satellites. The close extent is related to observation stations.

\subsection{PDOP without altimeter aiding}

In order to determine the user height CAPS barometric altimetry is comprised of some reference stations collecting local meteorological parameters. Meanwhile CAPS barometric altimetry technique is not available for some conditions with abrupt change whether. Therefore, there is difficulty in global navigation applications. Here we carry out daily PDOP change of constellation without altimeter aiding. Constellation with only four GEO satellites can't be used to realize navigation. We analyze improvement from $n(n=1,2,3,4)$ SIGSO satellites on CAPS constellation. Corresponding daily PDOP change is calculated. Here Fig. 6 displays daily PDOP change of CAPS constellation without altimeter aiding. 


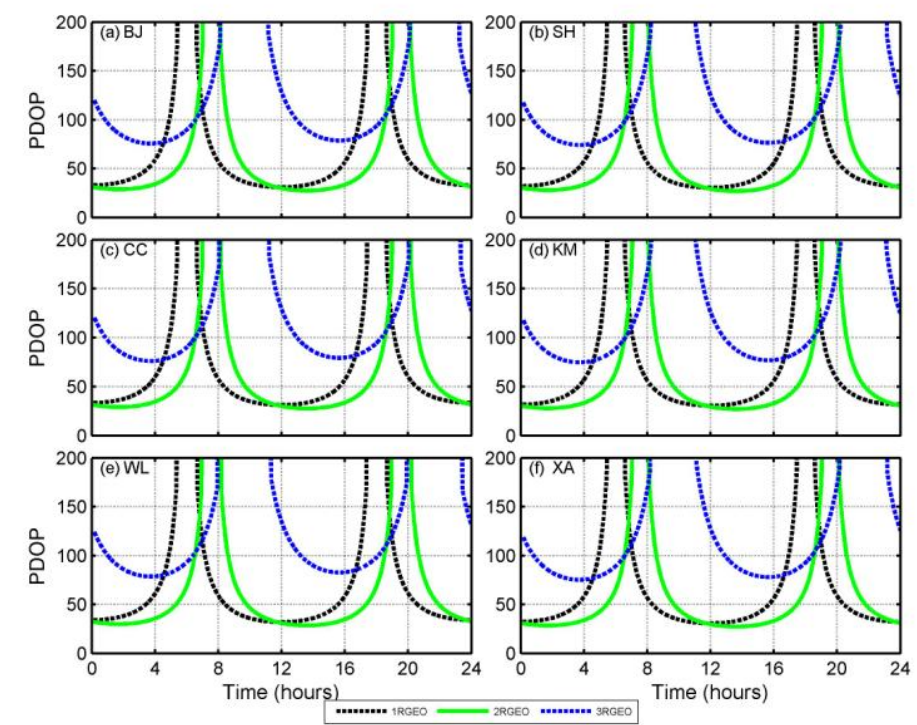

Fig. 6 Daily PDOP change of constellation with n SIGSO satellites

$$
(n=1,2,3) \text { without altimeter aiding }
$$

When 4 SIGSO satellites are used to construct navigation constellation, PDOP value in above stations exceeds 1000. Fig. 6 doesn't show this case. It can be seen that the user can be realized to navigation with poor precision in about half a day. From the figure, navigation and positioning is impossible in whole day on above stations. Meanwhile daily PDOP change with 1 SIGSO satellites is close to that with 2 SIGSO satellites. When 3 SIGSO satellites are used daily PDOP change will become worse.

\section{CONCLUSIONS}

Inclined-orbit operations can obviously extend the operational lifetime of GEO communication satellites. The SIGSO satellites from GEO satellites are smoothly applied to communication functions after adjusting satellite antenna coverage. Different from the GEO communication satellites, tacking mode for ground antenna should be regulated in order to utilize these satellites for obvious variation scale of azimuth and elevation angles exists in SIGSO satellites. Additionally, polarization regulation should be performed if satellites adopt linear polarization antenna. For navigation applications, navigation constellation can be constructed with low cost in a short period. The Doppler frequency shift can be countered by the frequency pre-bias technique. After processing carrier phase measurements, CAPS user can make velocity measurements. Combining with altimeter technique, improvement and deterioration is found in daily PDOP change. Because the GEO satellites locate at the equatorial plane it is impossible that navigation is performed in constellation with only GEO satellites. However, with SIGSO satellites, better navigation performance can be realized in about half a day. It is important how to effectively use SIGSO satellites not only with altimeter aiding but also without altimeter aiding, and detailed analysis will be included in an extended work in future.

Acknowledgements. The authors would like to thank Profs. G Ai, H Shi, Y Han and Q Qiao, who directed us in applied study in the GEO satellite in end of life. The project is supported by the National Basic Research and Development Program of China (Grant No. 2007CB815501) and the Young Researcher Grant of National Astronomical Observatories, Chinese Academy of Sciences. 


\section{REFERENCES}

Agrawal B. (1986) Design of geosynchronous spacecraft. Prentice-Hall, Englewood Cliffs, NJ.

Ai G, Sheng P, Du J, Zheng Y, Cai X, Wu H, Hu Y, Hua Y, Li X. (2009) Barometric altimetry system as virtual constellation applied in CAPS. Sci China Ser G-Phys Mech Astron, Vol. 52, No. 3, 376-383.

Ai G, Shi H, Wu H, Yan Y, Bian Y, Hu Y, Li Z, Guo J, Cai X. (2008) Positioning system based satellite communication and Chinese Area Positioning System (CAPS). Chin $J$ Astron Astrophys, Vol. 8, No. 6, 611-630.

Atia A, Day S, Westerlund L. (1990) Communications satellite operation in inclined orbit 'The Comsat Maneuver'. In: AIAA International Communication Satellite Systems Conference and Exhibit, 13th, Los Angeles, CA, Mar. 11-15, 1990, Technical Papers. Part 2 (A90-25601 09-32). Washington, DC, American Institute of Aeronautics and Astronautics, p. 452-455.

Bauer R, Krawczyk R, Irwin D, et al. (2002) Extending ACTS operations through a university-based consortium. Space Commun, Vol. 18, No. 1-2, 7-12.

Han Y, Ma L, Qiao Q, Yin Z, Shi H, Ai, G. (2009) Functions of retired GEO communication satellites in improving the PDOP value of CAPS. Sci China Ser G-Phys Mech Astron, Vol. 52, No. 3, 423-433.

Jwo D. (2001) Efficient DOP calculation for GPS with and without altimeter aiding. J Navig, Vol. 54, No. 2, 269-279.

Kaplan E, Hegarty C. (2006) Understanding GPS: Principles and Applications. 2nd ed. Artech House, Boston.

Li Y, Hao H. (1996) Satellite communication geosynchronous orbit with small inclination. Chin Space Sci Tech, Vol. 16, No. 3, 22-28.

Ma L, Hu C, Han Y, Qiao Q, Pei J. (2010) Simulation analysis of polarization matching for APSTAR-1 satellite in CAPS. J Syst Simul, Vol. 22, No. 10, 2446-2449.

Ma L, Jing Y, Ji H, Zhang L. (2011) The evolvement analysis of GEO satellite orbit in end of life based on STK software. Astron Res Tech (in press)

Ma L, Wang M, Zhang L, Ji H. (2011) Mathematical aspects of CAPS horizontal position error. Adv Space Res. (under reviewing)

Shi H, Ai G, Han Y, Ma L, Chen J, Geng J. (2009) Multi-life cycles utilization of retired satellites. Sci China Ser G-Phys Mech Astron, Vol. 52, No. 3, 323-327.

Shi H, Sun X and Li Z. (2009) Principle of transponder satellite navigation. Science Press, Beijing.

Wu H, Bian Y, Lu X, Li X, Wang D. (2009) Time synchronization and carrier frequency control of CAPS navigation signals generated on the ground. Sci China Ser G-Phys Mech Astron, Vol. 52, No. 3, 393-401.

Zhang R. (1998) Dynamics and control of satellite orbit and attitude. Beihang University Press, Beijing.

Received: 2011-02-08,

Reviewed: 2011-05-14, by A. Drożyner,

Accepted: 2011-05-29. 